using any standard fluorescence scanner.

Fluorescence is not the only detection system on offer in kit arrays. The range of chemiluminescence-based TransSignal protein-domain arrays from Panomics in Redwood City, California, is expanding, with the addition of $\mathrm{SH} 2$ and $\mathrm{WW}$ domains. The FASTQuant kit from Schleicher and Schuell in Keene, New Hampshire, combines traditional enzyme-linked immunosorbent assay (ELISA) methodology with the power of multiplexing. Antibodies to nine different human cytokines are incorporated into an array on the company's FASTSlides - nitrocellulosecoated glass slides - and 64 arrays are arranged in a microtitre plate footprint. The company plans to release six FASTQuant kits for human and mouse cytokines, angiogenesis factors and human chemokines this year.

\section{Adding content}

One bottleneck that chip manufacturers face is content. "Getting an antibody against every protein in the human proteome is a gigantic task, more so than sequencing the genome. This is not something that will happen in the next decade," says Jan van Oostrum, head of proteome sciences in functional genomics at Novartis in Basel, Switzerland.

Increasing the content is a matter of competitive advantage for Molecular Staging in New Haven, Connecticut, which uses protein chips in their work with biotech and pharma companies to identify biomarkers for diseases and help reanalyse promising drugs that, although highly effective in some patients, failed clinical trials because of unacceptable side-effects in others. "As soon as we see that a pathway is upregulated or downregulated we gain a mechanistic understanding of the disease, its progression, and patients' response to drugs," says Peter Fuller, senior vice-president of business development. "We work constantly to increase content - every four months we validate a new chip." The company's antibody-based chips use a signal-amplification technology developed at Yale University, New Haven. DNA circles attached to the detector proteins are replicated to produce a long DNA molecule that can be detected by attachment of large numbers of small detector molecules. Molecular Staging is collaborating with Eli Lilly to identify biomarkers for sepsis, a potentially deadly reaction to a blood infection that develops so rapidly that physicians find it difficult to diagnose quickly enough.

\section{Multiplexing in solution}

The challenges of constructing solid-surface arrays holding thousands of proteins with different properties are fuelling interest in protein-interaction assays in solution. Suspension-bead assays are particularly flexible, and can be adapted to both proteins and nucleic acids. The Bio-Plex system from Bio-Rad Laboratories in Hercules, California, uses Luminex's bead-based xMAP technology (see Nature 422, 917-923; 2003), as does the LiquiChip system from Qiagen Instruments in Hombrechtikon, Switzerland. For protein analysis, Bio-Plex uses antibodies from Cell Signaling Technologies in Beverly, Massachusetts, to offer ready-togo cytokine assays, and assays for phosphorylated signal transduction molecules, as well as customized arrays. Suspension-bead arrays are flexible enough to tackle any sort of protein-ligand interaction by simply coupling the required proteins or ligands to different bead populations. Luminex beads, for example, enable simultaneous quantitation of up to 100 different biomolecules in a single microplate well.

Another versatile platform is that of ACLARA Biosciences in Mountain View, California, which can support gene-expression and protein assays run side-by-side on the same sample. Detection is by proprietary eTAG reporter molecules, which are coupled to the ligands (for protein analysis these are usually antibodies), with a different eTAG for each ligand.Labelled ligands are applied to the sample in microtitre plates, or tissue sections on slides, and if the ligand binds to its target, the tag is released. Released tags are resolved, identified and quantified by capillary electrophoresis. An additional tacophoresis step concentrates the tags, taking detection sensitivity into the attomolar range. "It is possible to detect just 10 molecules," says Sharat Singh, chief technology officer at ACLARA. "In testing patient samples, we can predict whether a patient will respond or not by analysing a few analytes related to the mechanism of action of the drug and the biology of the specific target in the tumour."

The 3D HydroArray platform from Biocept in Carlsbad, California, uses a proprietary hydrogel polymer that is $95 \%$ water,

\title{
A NATURAL AFFINITY
}

How proteins interact with each other has become a key issue in drug discovery and development. Once a target for a drug is identified, it is also important to define how strongly it binds to other biomolecules to obtain information about selectivity. Biacore of Uppsala, Sweden, has been producing affinity-based sensor chips and instrumentation for protein-interaction analysis

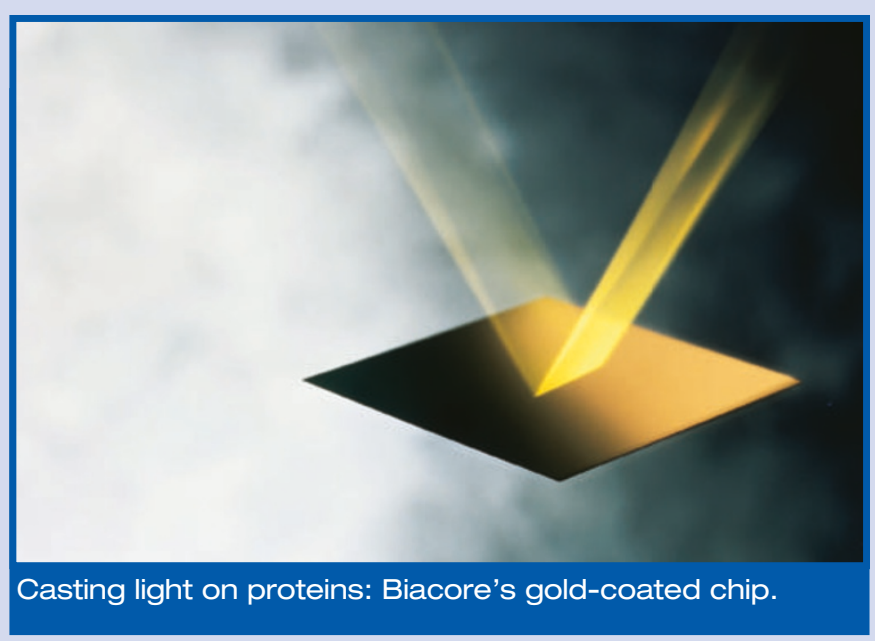

for more than a decade. The company's core technology is surface plasmon resonance (SPR), using a gold-coated glass chip onto which an array of protein-binding samples is immobilized. When the chip is illuminated, the interaction results in mass changes in the aqueous layer close to the sensor chip, that directly correlate to the refractive-index change. When molecules in the test solution bind to, or dissociate from, the immobilized protein, the refractive index rises and falls and a change is detected. Plotting the response against time allows continuous, real-time monitoring of interacting molecules. Because there is no need to label targets with fluorescent or radioactive labels, molecules can be studied in a near-native state, and the binding data closely reflect in vivo behaviour. Biacore has also developed the technology towards direct measurement of small-molecule binding to protein, for lead optimization in drug discovery, says Stephan Lofas, chief scientific officer. The company markets a variety of SPR instruments to suit different needs, from the highthroughput lab that requires unattended, fully automated runs for up to 384 samples at a time, to the smaller laboratory where researchers want to study a variety of different molecules.

The 8500 Affinity Chip Analyzer from Applied Biosystems, developed in conjunction with HTS Biosystems in Hopkinton, Massachusetts, is based on grating-coupled SPR technology. "If you are interested in target discovery, this system provides massively parallel detection of hundreds of binding events per analysis - thousands per day," says Enrico Picozza, chief technology officer at HTS Biosystems. 